\title{
Fundamental limitations on Earth-like planet detection with extremely large telescopes
}

\author{
C. Cavarroc ${ }^{1}$, A. Boccaletti ${ }^{1}$, P. Baudoz ${ }^{1}$, T. Fusco ${ }^{2}$, and D. Rouan ${ }^{1}$ \\ 1 LESIA, Observatoire de Paris Meudon, 5 Pl. J. Janssen, 92195 Meudon, France \\ e-mail: celine.cavarroc@obspm.fr \\ 2 ONERA, BP 52, 29 avenue de la Division Leclerc, 92320 Châtillon Cedex, France
}

Received 26 July 2005 / Accepted 20 September 2005

\begin{abstract}
We analyse the fundamental limitations for the detection of extraterrestrial planets with Extremely Large Telescopes. For this task, a coronagraphic device combined with a very high order wavefront correction system is required but is not sufficient to achieve the $10^{-10}$ contrast level needed to detect an Earth-like planet. The stellar residuals left uncorrected by the wavefront correction system need to be calibrated and subtracted. We consider a general model including the dynamic phase aberrations downstream of the wavefront correction system, the static phase aberrations of the instrument and some differential aberrations provided by the calibration unit. A rather optimistic case of a filled circular pupil and of a perfect coronagraph is assumed. As a result of the analytical study, the limitation is found to mostly come from the static aberrations. We confirm this result using numerical simulations and evaluate the requirements in terms of phase aberrations to detect Earth-like planets with Extremely Large Telescopes.
\end{abstract}

Key words. techniques: high angular resolution - instrumentation: adaptive optics - stars: imaging - stars: planetary systems

\section{Introduction}

Direct detection of Earth-like planets is a challenging issue, since a contrast of $10^{-10}$ must be reached at a close angular distance to a bright star. The characterization of exoplanets constitutes the main objective of several projects, whether from the ground or from space (Darwin, TPF-I and TPF-C for instance). Among ground-based projects, Extremely Large Telescopes (ELTs) are very promising to significantly improve the angular resolution. To date, several projects are considered such as the Giant Magellan Telescope (Johns et al. 2004), the Thirty Meter Telescope (Nelson et al. 2005), Euro50 (Andersen et al. 2003) and the $100 \mathrm{~m}$ OWL telescope (Gilmozzi 2004).

To detect Earth-like planets, the use of a coronagraph combined with an adaptive optics (hereafter AO) system is necessary but not sufficient. In the presence of atmospheric turbulence we cannot expect a contrast greater than $\sim 10^{-6}$ even with a high-order AO (whatever the coronagraphic system). Assuming an optimistic error budget for the AO, the Strehl ratio can be as large as $98 \%$ (see Sect. 3). Thus, the coronagraph is fed with an imperfect wavefront. Two percent of the stellar light still escapes the coronagraph and is spread in a speckled halo.

To enhance the contrast, a second step is mandatory to suppress this speckle noise. In this paper, we consider that the instrument provides a good calibration and that the speckle attenuation is achieved with a simple subtraction of two images (one of which shows the planet while the other does not). Due to the variability of the atmospheric phase distortion, the calibration must be done simultaneously. Here, for sake of generality we do not assume any particular system of calibration but several proposals were made in the past which take advantage of the planet properties like its spectral signature (Racine et al. 1999; Marois et al. 2000), its polarization (Baba \& Murakami 2003) or its coherence with respect to the star (Guyon 2004). The differential spectral imaging was already implemented in TRIDENT (Marois et al. 2005) and NACO-SDI (Close et al. 2005) and is also selected for the VLT Planet Finder (Beuzit \& et al. 2004).

Section 2 presents the formalism based on a simple model. Numerical simulations (Sect. 3) were done to confirm the analytical results and to assess the sensitivity to the various phase aberrations. Important conclusions are drawn in Sect. 5.

\section{Formalism}

\subsection{Assumptions}

The following assumptions are very general and mostly optimistic with respect to the performance of the state-of-the-art instruments.

We consider a circular unobstructed pupil without segmentation. The coronagraph is assumed perfect and is modeled by the subtraction of a perfect pupil from the actual aberrated one 
(see Eqs. (1) and (3)) in order to remove the diffraction. It is achromatic, the star is perfectly centered and the Lyot stop is perfectly aligned.

Several coronagraphs that provide a total extinction of an on-axis unresolved point source with a zero aberration system have been already proposed (Roddier \& Roddier 1997; Rouan et al. 2000; Baudoz et al. 2000; Kuchner \& Traub 2002). To find the most suitable type of coronagraph it will be necessary to take into account the pupil defects of actual ELTs (segmentation, obstruction, cophasing errors between segments,...).

We consider the following contributions to the phase errors in the wavefront: residual phase from AO correction $(\phi)$ and static aberrations $\left(\delta_{\mathrm{C}}\right)$ in the instrument upstream of the coronagraph. Consideration of this second type of aberration is required to be realistic, even if they are not totally static but "quasi static" with a lifetime from a few seconds to a few minutes (Marois et al. 2003).

With these notations and assuming a perfect coronagraph, the complex amplitude $A_{1}$ in the pupil plane can be written as:

$A_{1}=\Pi\left(\sqrt{E_{C}}-\mathrm{e}^{\mathrm{i}\left(\phi+\delta_{\mathrm{C}}\right)}\right)$

with $\Pi$ the entrance pupil, $E_{\mathrm{C}}=\mathrm{e}^{-\sigma_{\phi+\delta_{\mathrm{C}}}^{2}}$ the instantaneous coherent energy and $\sigma_{\phi+\delta_{\mathrm{C}}}^{2}$ the spatial variance of the AO residual phase and of the static aberration.

Assuming a high Strehl ratio (more than 95\%) i.e. considering that wavefront errors are small (a few nanometers or tens of nanometers rms), a third order expansion can be made and is justified in the next section. We can approximate $A_{1}$ with:

$A_{1}=\Pi\left(-\frac{\sigma_{\phi+\delta_{\mathrm{C}}}^{2}}{2}-\mathrm{i}\left(\phi+\delta_{\mathrm{C}}\right)+\frac{\left(\phi+\delta_{\mathrm{C}}\right)^{2}}{2}+\mathrm{i} \frac{\left(\phi+\delta_{\mathrm{C}}\right)^{3}}{6}\right)$.

\subsection{Instantaneous residual intensity}

We consider here two images taken simultaneously using two channels downstream of the coronagraph to calibrate the residual speckle pattern. A potential high source of mismatch in between the two images is the non-common path wavefront aberration since the light goes through two differential optical paths. So, we will consider a "reference" channel having only $\phi$ and $\delta_{\mathrm{C}}$, the amplitude of which is described by Eq. (1) and a second channel which, in addition to $\phi$ and $\delta_{\mathrm{C}}$, includes a non-common path aberration $\delta_{\mathrm{NC}}$ with an amplitude defect of the same order as $\phi$ and $\delta_{\mathrm{C}}$. Its amplitude in the pupil plane can be written by:

$A_{2}=\Pi\left(\sqrt{E_{\mathrm{C}}}-\mathrm{e}^{\mathrm{i}\left(\phi+\delta_{\mathrm{C}}\right)}\right) \mathrm{e}^{\mathrm{i} \delta_{\mathrm{NC}}}$

As for Eq. (1) a third order expansion gives:

$A_{2}=A_{1}+\Pi\left(-\mathrm{i} \frac{\sigma_{\phi+\delta_{\mathrm{C}}}^{2}}{2} \delta_{\mathrm{NC}}+\left(\phi+\delta_{\mathrm{C}}\right) \delta_{\mathrm{NC}}+\mathrm{i} \frac{\left(\phi+\delta_{\mathrm{C}}\right)^{2} \delta_{\mathrm{NC}}}{2}\right)$

The intensity $I_{2}$ in the detector plane corresponds to the square modulus of the Fourier transform of $A_{2}$ (neglecting terms beyond third order). We will denote Fourier transforms by a hat accent. The star $\star$ represents the convolution operator.

$$
\begin{aligned}
I_{2} & =\left|\widehat{A_{2}}\right|^{2} \\
& =I_{1}+2 \mathfrak{J}\left[\left(\widehat{\Pi} \star\left(\widehat{\phi}+\widehat{\delta_{\mathrm{C}}}\right)\right) \cdot\left(\widehat{\Pi} \star\left(\widehat{\phi}+\widehat{\delta_{\mathrm{C}}}\right) \star \widehat{\delta_{\mathrm{NC}}}\right)^{*}\right]
\end{aligned}
$$

with * the complex conjugation and $\mathfrak{J}[$ ] the imaginary part of the complex quantity. $I_{1}$ is the intensity of the reference image $\left(I_{1}=\left|\widehat{A_{1}}\right|^{2}\right)$.

Subtracting $I_{1}$ from $I_{2}$, we obtain:

$$
\begin{aligned}
I_{\mathrm{NC}}=I_{2}-I_{1}= & 2 \mathfrak{J}\left[(\widehat{\Pi} \star \widehat{\phi}) \cdot\left(\widehat{\Pi} \star \widehat{\phi} \star \widehat{\delta_{\mathrm{NC}}}\right)^{*}\right] \\
& +2 \mathfrak{J}\left[(\widehat{\Pi} \star \widehat{\phi}) \cdot\left(\widehat{\Pi} \star \widehat{\delta_{\mathrm{C}}} \widehat{\delta_{\mathrm{NC}}}\right)^{*}\right] \\
& +2 \mathfrak{J}\left[\left(\widehat{\Pi} \star \widehat{\delta_{\mathrm{C}}}\right) \cdot\left(\widehat{\Pi} \star \widehat{\phi} \star \widehat{\delta_{\mathrm{NC}}}\right)^{*}\right] \\
& +2 \mathfrak{J}\left[\left(\widehat{\Pi} \star \widehat{\delta_{\mathrm{C}}}\right) \cdot\left(\widehat{\Pi} \star \widehat{\delta_{\mathrm{C}}} \widehat{\delta_{\mathrm{NC}}}\right)^{*}\right]
\end{aligned}
$$

$I_{\mathrm{NC}}$ refers to the instantaneous residual intensity after subtraction.

$\Pi, \phi, \delta_{\mathrm{C}}$ and $\delta_{\mathrm{NC}}$ are real, so their Fourier transforms are Hermitian: their real part is even and their imaginary part is odd. Thus, the resulting image $I_{\mathrm{NC}}$, which is made only with imaginary parts, is antisymmetric (providing the expansion is stopped at the third order).

Equation (6) has only terms of the third order. It is therefore obvious that the third order expansions in Eqs. (2) and (4) are required to obtain $I_{\mathrm{NC}} \neq 0$. All terms that classically dominate imaging are removed in the 2-channel subtraction process. Indeed, an image made with a perfect coronagraph has neither residual constant nor first order terms, as developed in Boccaletti et al. (2002) and Bloemhof (2004) in the case of the Four Quadrant Phase Mask. Moreover, the interest of coronagraphic image subtraction lies in removing terms of degree two, which are studied in Perrin et al. (2003) and Sivaramakrishnan et al. (2002) in a direct imaging case.

The terms of Eq. (6) vary linearly with $\widehat{\delta_{\mathrm{NC}}}$. Because of the linearity of the Fourier transform, a linear variation in $\delta_{\mathrm{NC}}$ induces a linear variation in the level of the residual intensity. Each term of Eq. (6) is shown in Fig. 1 for a single instantaneous image.

The approximation (third order expansion) of Eqs. (2) and (4) is valid when the aberrations $\phi, \delta_{\mathrm{C}}$ and $\delta_{\mathrm{NC}}$ have similar orders of magnitudes. In actual cases, $\delta_{\mathrm{NC}}$ will differ by 1 or 2 orders of magnitude which changes the order of the expansion but Eq. (6) will remain unchanged (as confirmed in Fig. 5).

\section{Numerical simulations}

We carried out numerical simulations to confirm the analytical study. Simulations were performed for a thirty meter telescope, using arrays of $2048 \times 2048$ pixels with a sampling of $3.6 \mathrm{~cm}$ per pixel at the pupil plane and for a wavelength $\lambda=1.63 \mu \mathrm{m}$. We considered a filled circular aperture.

The atmospheric phase screens are generated with a tool based on the approach first introduced by Rigaut (Rigaut et al. 1998; Jolissaint \& Veran 2002). Analytical expressions of the spatial power spectral density (PSD) of the residual phase are obtained for various errors affecting an AO system (fitting, aliasing, temporal, noise, anisoplanatism ...). The resulting global AO PSD (sum of the individual PSD of each error 


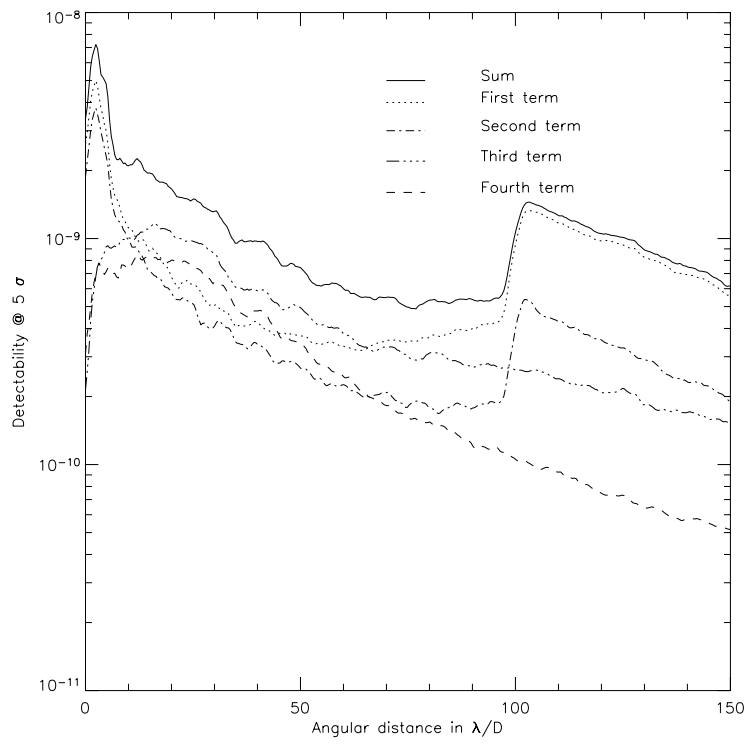

Fig. 1. Radial profile of the standard deviation (azimuthally averaged) of each term in Eq. (6) for an instantaneous image (1 speckle pattern). Simulation parameters are given in Sect. 3.

source) is used to compute AO corrected phase screens (independent realizations of the global AO PSD). Recent works (Conan et al. 2005) allowed us to include a correct model of AO closed loop temporal and noise effects (assuming an intregator law) as well as the differential refraction effects.

In the following, we consider a Shack-Hartmann wavefront sensor with 200 actuators across the telescope diameter. The seeing is $0.75 \operatorname{arcsec}$ at $\lambda=0.5 \mu \mathrm{m}$ and the outer scale is $20 \mathrm{~m}$. We assumed a 5th magnitude star and a loop frequency of $2.5 \mathrm{kHz}$. The AO PSD includes fitting errors, servo lag and the photon noise on the wavefront sensor. These AO parameters provide a very good wavefront correction and a Strehl ratio of $98 \%$ which is a great challenge for ELTs.

The static phase aberrations $\delta_{\mathrm{C}}$ and $\delta_{\mathrm{NC}}$ are described with a PSD which varies as $f^{-2}$ (with $f$ the spatial frequency) as is usually the case for standard optical components (Duparré et al. 2002). In the case of high contrast imaging with a coronagraph, special care is needed to reduce the impact of low spatial frequencies. We therefore assumed that the PSD at low frequencies was already improved and that it is flat in the range $0<f<f_{\mathrm{C}} / 4$, with $f_{\mathrm{C}}$ the cut-off frequency of the AO defined by the number of actuators across the pupil. As a first guess we adopt an amplitude of $20 \mathrm{~nm} \mathrm{rms}$ and $0.1 \mathrm{~nm}$ rms for $\delta_{\mathrm{C}}$ and $\delta_{\mathrm{NC}}$ respectively. The impact of the amplitude and the PSD of the phase aberrations are further investigated in Sects. 3.3 and 3.4 .

\subsection{Short exposure image}

We first study a single short exposure coronagraphic image subtracted by a calibration image. Its theoretical expression is given by Eq. (6). Figure 2 shows the central part of an instantaneous simulated image after subtraction $\left(I_{\mathrm{NC}}=I_{2}-I_{1}\right)$. Positive speckles are white while negative ones are black. The intensity

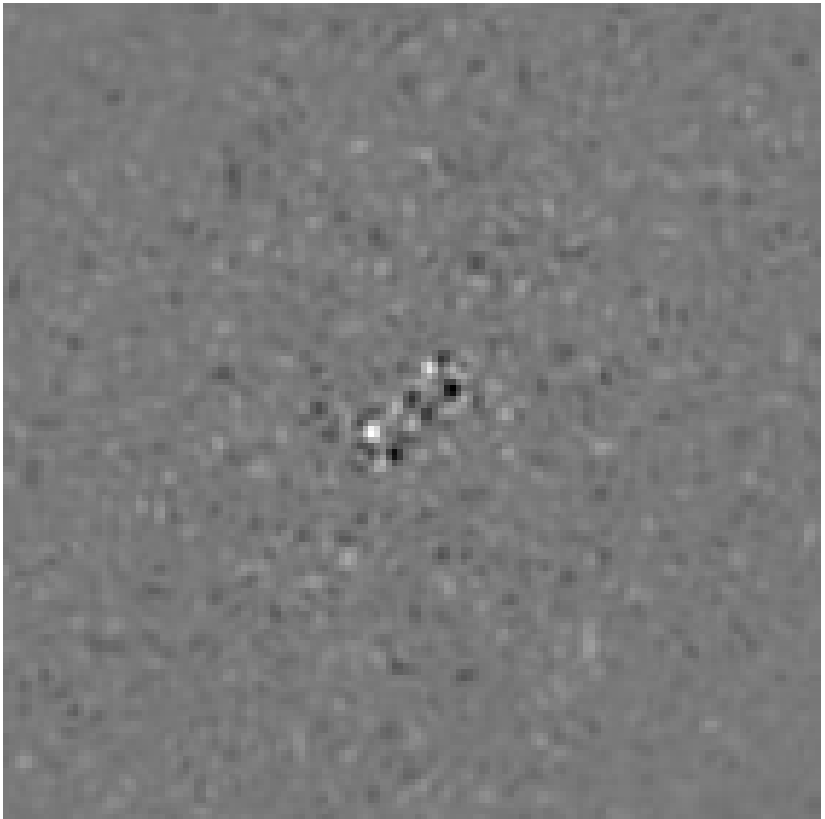

Fig. 2. Central part of an instantaneous image (fov $=54 \lambda / D)$. The antisymmetrical speckle pattern is shown. The simulation was done for $\delta_{\mathrm{C}}=20 \mathrm{~nm} \mathrm{rms}$ and $\delta_{\mathrm{NC}}=0.1 \mathrm{~nm} \mathrm{rms}$ and with a power square law spectrum truncated at $f_{\mathrm{c}} / 4$ ( $f_{\mathrm{c}}$ is the cut-off frequency of the AO system).

distribution in the focal plane is clearly anti-centrosymmetrical as expected from Eq. (6).

Unlike direct imaging, speckle pinning (Bloemhof et al. 2001) which appears when a phase defect is multiplied by the Fourier transform of the pupil $(\widehat{\Pi})$ does not occur here (see Eq. (6)) due to the coronagraph effect and the image subtraction. Thus, residual speckles are randomly distributed in the image and not only pinned on the rings of the point spread function.

Figure 3 compares radial profiles of the image before and after subtraction of a single exposure. The agreement between simulated and analytical profiles is perfect, indicating that the approximation we made in Sect. 2 is valid at this level of contrast.

\subsection{Convergence over time}

A long exposure image is generated by co-adding $N$ decorrelated instantaneous images while static aberrations remain unchanged. The final image delivered by the simulation is $\left\langle I_{\mathrm{NC}}\right\rangle$ where \langle\rangle denotes temporal averaging over the $N$ realizations. In the following, we analyze this simulation result in terms of the detectability of a point source. For that, we calculated the standard deviation in the image $\left\langle I_{\mathrm{NC}}\right\rangle$ as explained in Boccaletti (2004). This operation is termed $\sigma_{\theta}$ and is measured on a sample of pixels at equal distance to the star.

In Fig. 4, the radial profiles are calculated for increasing values of $N$ from a single exposure to $N=460$. This simulation shows that the detectability converges towards the standard deviation of the constant term of Eq. (6) (dashed line in Fig. 4):

$\sigma_{\theta}\left(\left\langle I_{\mathrm{NC}}\right\rangle\right)=\sigma_{\theta}\left(2 \mathfrak{J}\left[\left(\widehat{\Pi} \star \widehat{\delta_{\mathrm{C}}}\right) \cdot\left(\widehat{\Pi} \star \widehat{\delta_{\mathrm{C}}} \star \widehat{\delta_{\mathrm{NC}}}\right)^{*}\right]\right)$. 


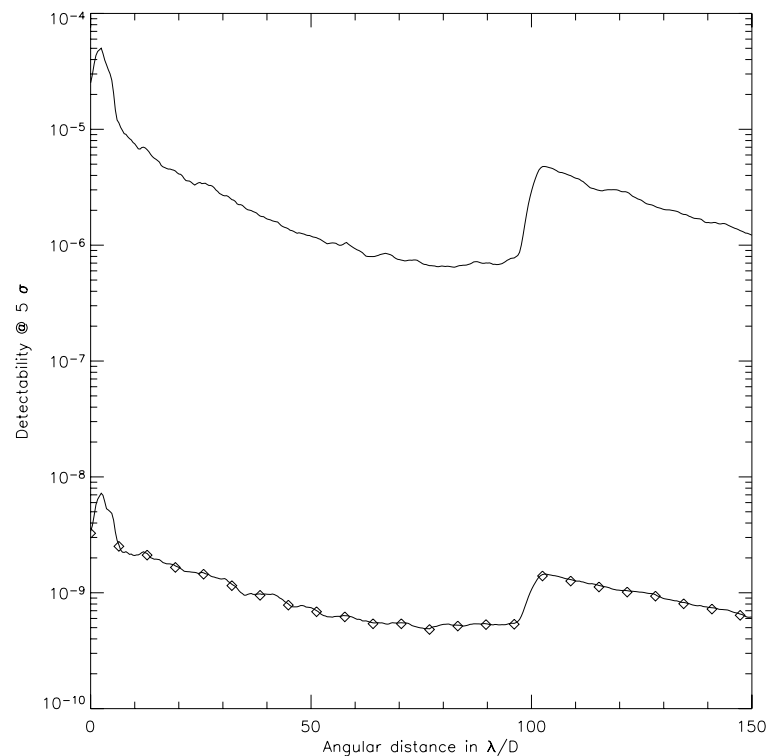

Fig. 3. Comparison between analytical (solid lines) and simulated curves (diamonds) calculated to achieve a $5 \sigma$ detection. The upper curve corresponds to the raw coronagraphic image $I_{1}$. The bottom curve corresponds to standard deviation of the instantaneous image after subtraction, $I_{\mathrm{NC}}$ (Eq. (6)). The static aberrations have the same amplitude and PSD as in Fig. 2.

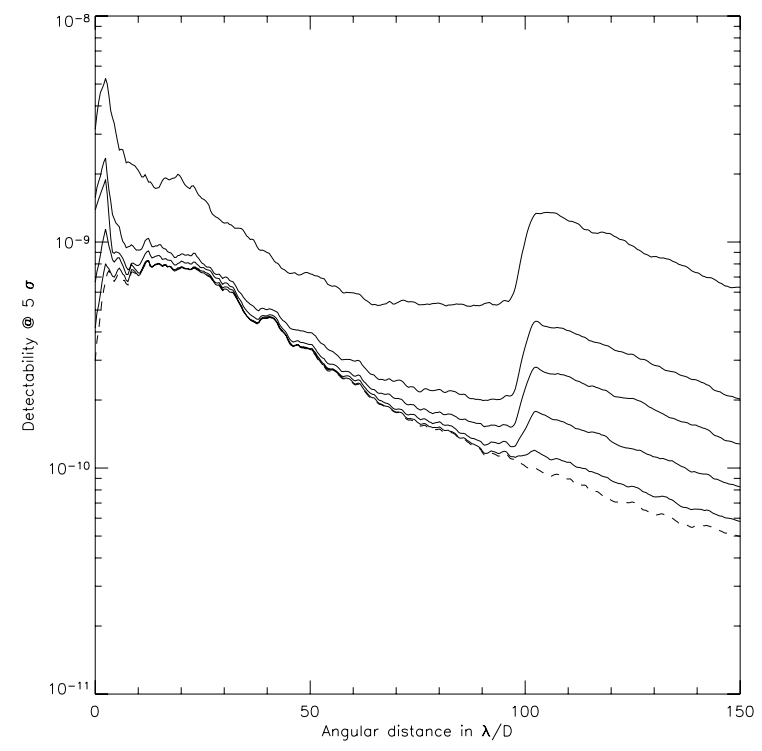

Fig. 4. Detectability at $5 \sigma$ as a function of the number of realizations $N$. The different solid lines represent the azimuthal standard deviation of the quantity $\left\langle I_{\mathrm{NC}}\right\rangle$ and are calculated with 9, 27, 90 and 460 decorrelated speckle patterns (from top to bottom respectively). The theoretical limit is derived from Eq. (7) (dashed line). The static aberrations have the same amplitude and PSD as in Fig. 2.

The other terms containing $\phi$ average to an azimuthally constant pattern over time when a large number of phase screens are simulated $(N \gtrsim 500)$. Therefore, the detectability only depends on the static aberrations upstream $\left(\delta_{\mathrm{C}}\right)$ and downstream $\left(\delta_{\mathrm{NC}}\right)$ the coronagraph and Eq. (7) gives the fundamental limit

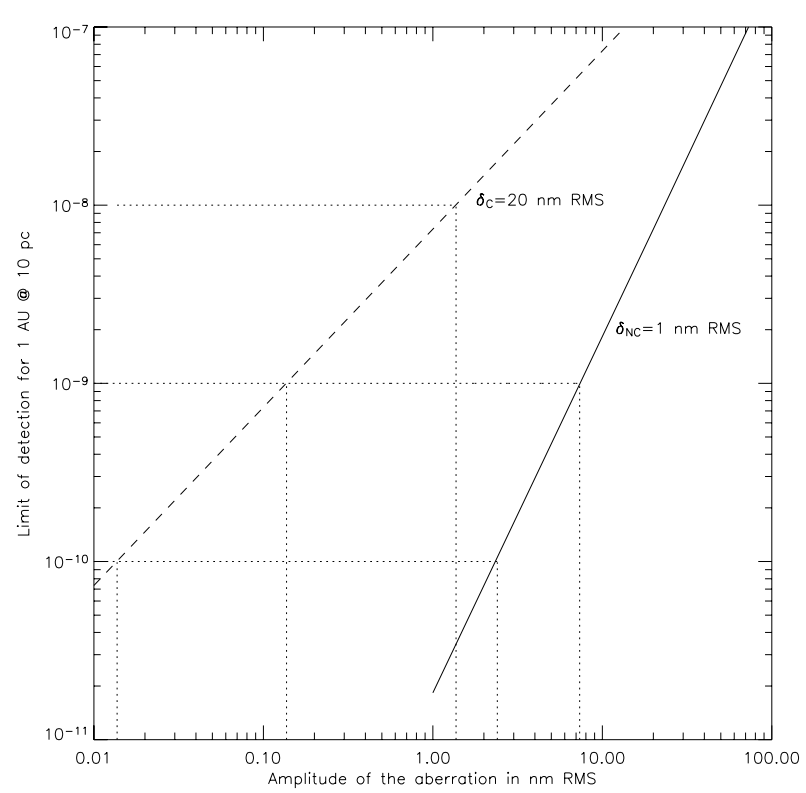

Fig. 5. Detectability at $5 \sigma$ for an angular separation of $9 \lambda / D$ corresponding to $1 \mathrm{AU}$ at $10 \mathrm{pc}$. The solid line stands for the impact of $\delta_{\mathrm{C}}$ when $\delta_{\mathrm{NC}}$ is set to $1 \mathrm{~nm}$ rms and the dashed line represents the impact of $\delta_{\mathrm{NC}}$ when $\delta_{\mathrm{C}}$ is set to $20 \mathrm{~nm}$ rms. The PSD of aberrations is identical to Fig. 2.

of contrast with the model we have considered. The detectability varies linearly with $\delta_{\mathrm{NC}}$ but quadratically with $\delta_{\mathrm{C}}$. In addition, it is clear that a simulation where the static aberrations are omitted leads to a complete averaging of the speckle pattern over time, which is unrealistic.

In the following we will only consider Eq. (7) as the fundamental limitation to assess the influence of $\delta_{\mathrm{C}}$ and $\delta_{\mathrm{NC}}$.

\subsection{Influence of static aberration amplitudes}

The influence of the amplitude of the static aberrations is shown in Fig. 5 for a radial distance in the field corresponding to a 1 AU orbit located at $10 \mathrm{pc}$. The impact of common path and non-common path static aberrations is clearly different. The dependence of the detectability varies quadratically with $\delta_{\mathrm{C}}$ and linearly with $\delta_{\mathrm{NC}}$ as expected from Eq. (7). Therefore, the influence of common static aberrations upstream of the coronagraph is more important.

To reach a contrast of $10^{-10}$ at $0.1^{\prime \prime}$ required to detect an Earth-like planet located at $10 \mathrm{pc}$, the non-common static aberrations downstream of the coronagraph must be controlled to $0.01 \mathrm{~nm}$ rms if common aberrations are set to $20 \mathrm{~nm}$ rms. Vice versa, one can tolerate non-common aberrations of $1 \mathrm{~nm}$ rms if the common aberrations upstream of the coronagraph are lowered to $3 \mathrm{~nm}$ rms. Common aberrations are dominant and must be reduced as much as possible.

Figure 6 shows the radial profile of the detectability at $5 \sigma$ for different amplitudes of $\delta_{\mathrm{NC}}$ assuming $\delta_{\mathrm{C}}$ is set to $20 \mathrm{~nm} \mathrm{rms}$ (a challenging but realistic value). The linearity is again shown here. 


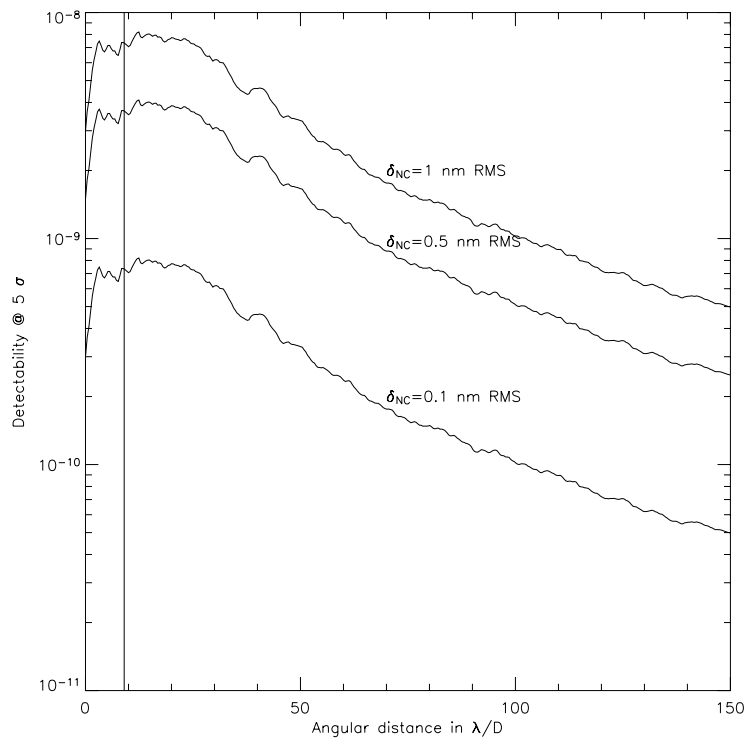

Fig. 6. Profiles of detectability at $5 \sigma$ with a common path aberration $\delta_{\mathrm{C}}$ fixed to $20 \mathrm{~nm}$ rms for several values of $\delta_{\mathrm{NC}}$. The vertical line shows the position of a $1 \mathrm{AU}$ orbit at $10 \mathrm{pc}$.

\subsection{Influence of static aberration PSDs}

Assuming the amplitudes of $\delta_{\mathrm{C}}$ and $\delta_{\mathrm{NC}}$ are set to 20 and $0.1 \mathrm{~nm} \mathrm{rms}$ respectively, we analyzed the influence of the Power Spectrum Density in Fig. 7. The solid line corresponds to our standard conditions, where the PSD of $\delta_{\mathrm{C}}$ and $\delta_{\mathrm{NC}}$ is flat between 0 and $f_{\mathrm{C}} / 4$ and then varies as $f^{-2}$ for $f>f_{\mathrm{C}} / 4$. The degradation is not dramatic if the PSD of $\delta_{\mathrm{NC}}$ varies as $f^{-2}$ at all frequencies (dashed line) which confirms the low sensitivity to this parameter. However, when the PSD of $\delta_{\mathrm{C}}$ is not flat at low frequencies (dotted line) the detectability decreases by a factor of 10 at small angular distances $(\sim 3 \lambda / D)$ and meets the solid line at $30 \lambda / D$. Better results are obtainable if the PSD of static aberrations can be made flat at all frequencies (dot-dashed and dot-dot-dot-dashed lines). A significant improvement of the detectability is achievable when for instance the PSD of $\delta_{\mathrm{C}}$ is flat even if that of $\delta_{\mathrm{NC}}$ is quadratic (dot-dashed line).

Again, the analysis of the PSD shows that $\delta_{\mathrm{C}}$ is the dominant source of degradation and that the low frequencies must be appropriately controlled to achieve a good starlight reduction.

\subsection{Photon noise and influence of the diameter}

Another fundamental limitation we have to take into account is the photon noise. It is important to know if the contrast level in Eq. (7) is achievable in a reasonable amount of time. We assumed a 5th magnitude G2V star at $10 \mathrm{pc}$ observed with a $30 \mathrm{~m}$ telescope, a spectral resolution of $\lambda / \Delta \lambda=5$ and an overall transmission of $5 \%$.

Figure 8 (left) gives the limit of detectability for several exposure times from 1 to $1000 \mathrm{~h}$ and compares it to the expression of Eq. (7) (solid line). The photon noise is included in the image $I_{1}$ and $I_{2}$ and therefore the detectability has a radial profile impressed by the distribution of the atmospheric turbulence residual $\phi$. As the integration time increases, the detectability

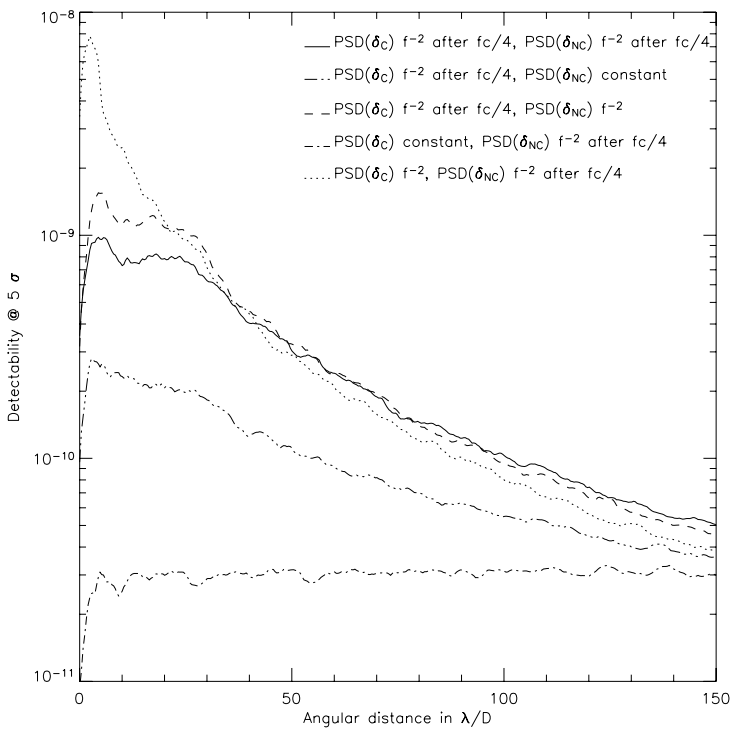

Fig. 7. Radial profile at $5 \sigma$ for several combinations of PSD. For each case, the amplitude of $\delta_{\mathrm{C}}$ and $\delta_{\mathrm{NC}}$ are set to 20 and $1 \mathrm{~nm} \mathrm{rms}$. The solid line stands for the standard condition we have considered in previous sections to evaluate the detectability.

level and its profile converge to the expression given in Eq. (7). A few hundreds of hours are required to achieve a $5 \sigma$ contrast of $8 \times 10^{-10}$ at a separation of $0.1^{\prime \prime}$.

A more favorable case is achieved with a $100 \mathrm{~m}$ telescope (Fig. 8 right) assuming static aberrations have the same amplitudes $\left(\delta_{\mathrm{C}}=20 \mathrm{~nm} \mathrm{rms}\right.$ and $\left.\delta_{\mathrm{NC}}=0.1 \mathrm{~nm} \mathrm{rms}\right)$ and a cut-off frequency of $f_{c} / 4$. The $\mathrm{AO}$ system has the same actuator density than for a $30 \mathrm{~m}$ telescope so the linear number of actuators is 667. In that situation, the influence of static aberrations is greatly relaxed and a contrast of $2 \times 10^{-10}$ is achievable in about $100 \mathrm{~h}$ at $0.1^{\prime \prime}$. The PSDs of static aberrations have the same integral for the $30 \mathrm{~m}$ and the $100 \mathrm{~m}$ telescopes but the cut-off frequency is 3 times larger $(83 \lambda / D$ instead of $25 \lambda / D)$ so that the amplitude of low frequencies is reduced and finally, the limit of detection is improved by a factor of 5 .

However, it is more realistic to consider that the static aberrations are located inside the instrument, the size of which does not depend on the telescope diameter. In that case, the cut-off frequency and the integral of the PSD are identical for the $30 \mathrm{~m}$ and the $100 \mathrm{~m}$ telescopes and the limit of detection is found to be $8 \times 10^{-10}$ as in Fig. 8 (left). Nevertheless, the integration time to achieve this contrast is about $100 \mathrm{~h}$ because the natural contrast of the PSF at $0.1^{\prime \prime}$ is improved with respect to the $30 \mathrm{~m}$ telescope (Fig. 9).

\section{Comparison with previous studies}

Several analytical studies were carried out in the past to assess the detectability of Earth-like planets with ELTs (Angel 2003; Lardiere et al. 2004; Chelli 2005). As shown in the previous section, the integration time depends on several parameters and in particular on the PSF contrast which quantifies the level of the residual light at a given angular separation on a short exposure. If the speckle noise is neglected and static aberrations are 

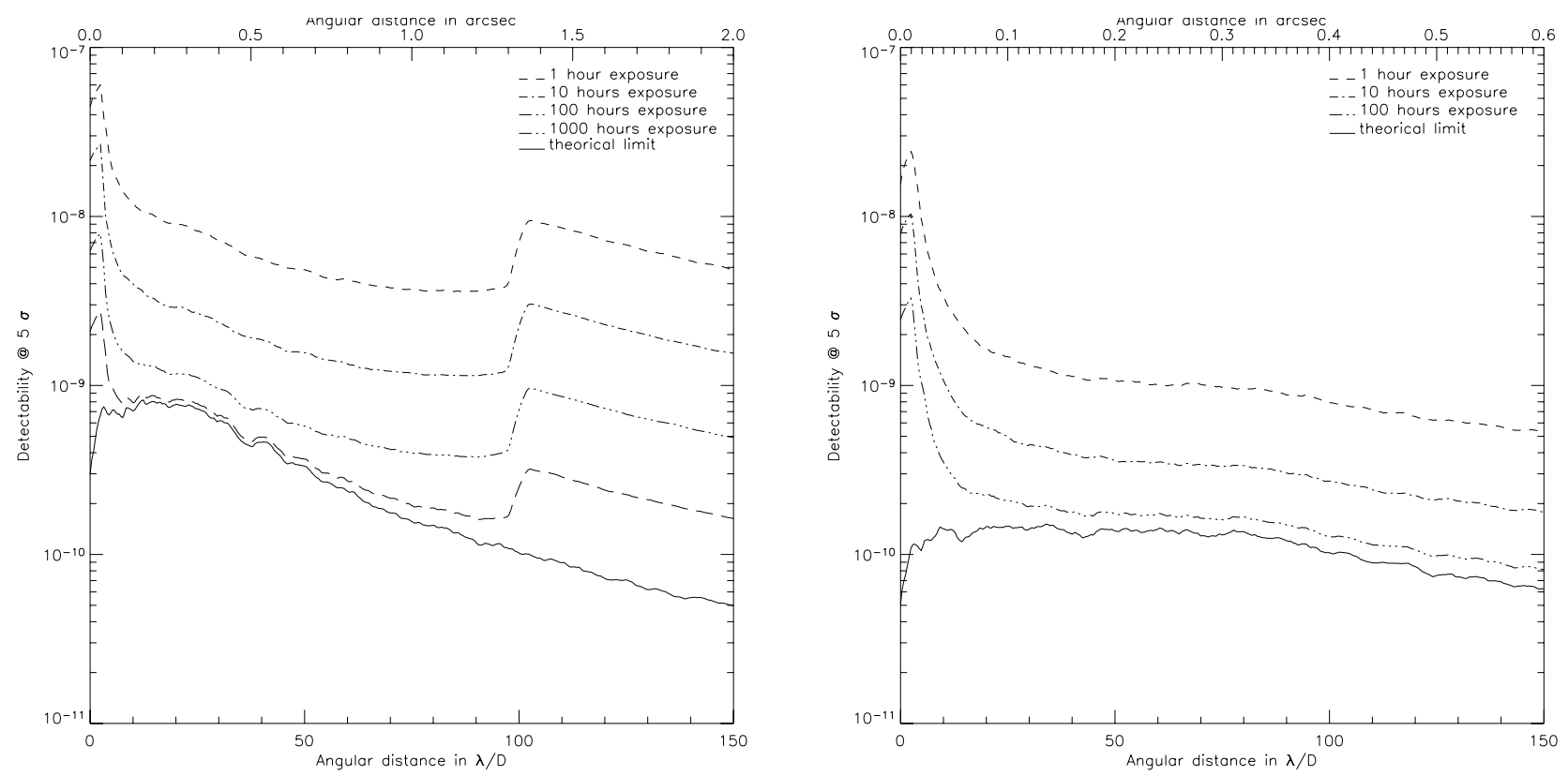

Fig. 8. Radial profile of the detectability at $5 \sigma$ for a $30 \mathrm{~m}$ telescope (left) and a $100 \mathrm{~m}$ telescope (right). The lower curve corresponds to the theoretical limit $\left(\delta_{\mathrm{C}}=20 \mathrm{~nm} \mathrm{rms}\right.$ and $\left.\delta_{\mathrm{NC}}=0.1 \mathrm{~nm} \mathrm{rms}\right)$ for an infinitely long exposure. Static aberrations scale with the diameter.

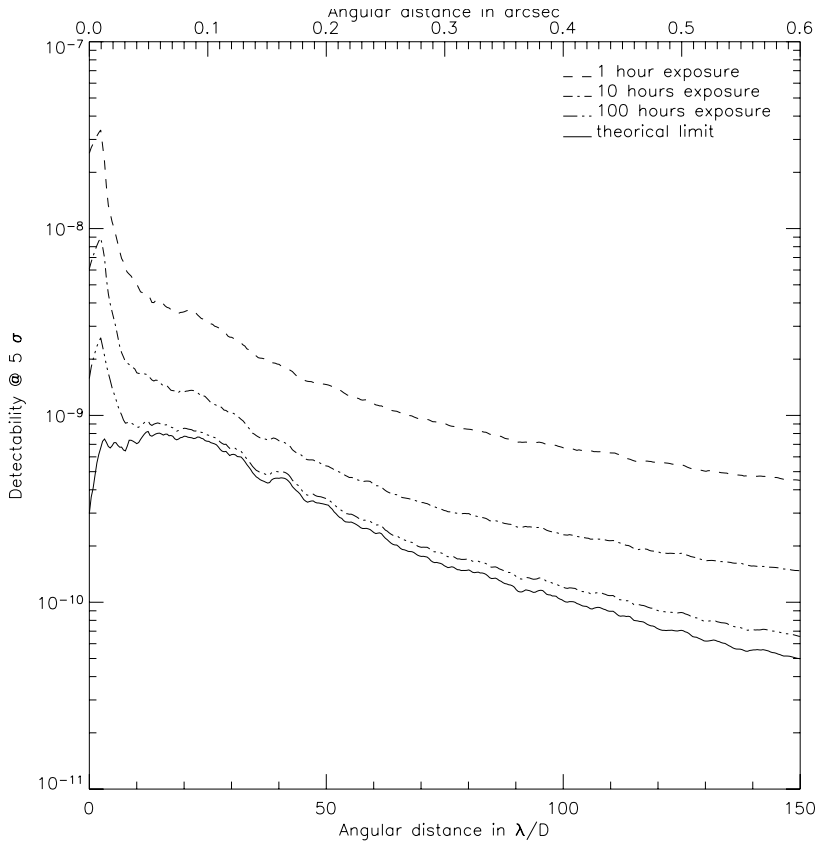

Fig. 9. Radial profile of the detectability at $5 \sigma$ for a $100 \mathrm{~m}$ telescope. Static aberrations are included in the instrument rather than on the telescope and are independent of the diameter. The lower curve corresponds to the theoretical limit $\left(\delta_{\mathrm{C}}=20 \mathrm{~nm} \mathrm{rms}\right.$ and $\delta_{\mathrm{NC}}=0.1 \mathrm{~nm}$ rms) for an infinitely long exposure.

omitted as in Lardiere et al. (2004), the signal to noise ratio is found to be only limited by the photon noise in the PSF halo. In that condition, the integration time varies linearly with the PSF contrast $C(\theta)$ where $\theta$ is the angular separation of interest. The critical parameter $C(\theta)$ is derived from the PSD of the residual phase left uncorrected by the AO system and therefore varies according to the different authors depending on the amplitude of the errors considered (servo lag, photon noise on the WFS, fitting errors, ...).

In Lardiere et al. (2004), this PSF contrast amounts to $5 \times 10^{-8}$ for a $100 \mathrm{~m}$ telescope which allows the detection of a $10^{-10}$ contrast in $3.5 \mathrm{~h}$ at $5 \sigma$ assuming a stellar flux of $10^{10} \mathrm{ph} / \mathrm{s}$. Similar performances were obtained by Chelli (2005) (where the speckle noise is treated correctly) and Angel (2003) who both adopted the same value of $C(\theta)$.

In our study, we found significantly longer integration times even with a $100 \mathrm{~m}$ telescope for which the static aberrations are set to a very low level (Fig. 8 right). This discrepancy originates from the performance of the AO system since our phase screen simulation yields a PSF contrast at $0.1^{\prime \prime}$ of $1.5 \times 10^{-6}$ for a $30 \mathrm{~m}$ telescope and $5 \times 10^{-7}$ for a $100 \mathrm{~m}$ which corresponds in the Lardiere et al. model to integration times of $100 \mathrm{~h}$ and $35 \mathrm{~h}$ respectively. The numerical simulation including photon noise (Sect. 3.5) and a more realistic model of the phase aberrations is even less favorable and predicts an integration time of about $100 \mathrm{~h}$ on a $100 \mathrm{~m}$ telescope to achieve a contrast of $2 \times 10^{-10}$ at $5 \sigma$.

From this rough comparison, it is clear that other than the problem of static aberrations, the potential of ELTs to detect Earth-like planets mostly resides in the performance of the AO system.

\section{Conclusion}

In this study, we considered an actual system, taking into account some defects due to the $\mathrm{AO}$ residuals $\phi$, the common path aberrations $\delta_{\mathrm{C}}$ between the $\mathrm{AO}$ system and the coronagraph and the non-common path aberrations $\delta_{\mathrm{NC}}$ downstream 
of the coronagraph. From that simple but realistic model we draw important conclusions:

- An actual system has $\phi>\delta_{\mathrm{C}}>\delta_{\mathrm{NC}} \neq 0$. Any study where $\delta_{\mathrm{C}}$ is neglected leads to unrealistic results where the variance of the residual intensity necessarily converges to 0 for an infinitely long exposure.

- The fundamental limit for an infinitely long exposure is constant and is given by:

$$
\sigma_{\theta}\left(\left\langle I_{\mathrm{NC}}\right\rangle\right)=\sigma_{\theta}\left(2 \mathfrak{J}\left[\left(\widehat{\Pi} \star \widehat{\delta_{\mathrm{C}}}\right) \cdot\left(\widehat{\Pi} \star \widehat{\delta_{\mathrm{C}}} \star \widehat{\delta_{\mathrm{NC}}}\right)^{*}\right]\right)
$$

- This detectability has a quadratic dependence in $\delta_{\mathrm{C}}$ and a linear dependence in $\delta_{\mathrm{NC}}$ but does not depend on the AO performance because $\phi$ averages to 0 over time.

- Improvement of the detectability can be obtained if the power spectrum density of the static phase aberrations is decreased, especially at low and mid frequencies.

- To achieve a $5 \sigma$ contrast of $10^{-9}$ with a $30 \mathrm{~m}$ telescope when the common aberrations are $20 \mathrm{~nm} \mathrm{rms}$, the calibration must be performed at a level of $\delta_{\mathrm{NC}}=0.1 \mathrm{~nm} \mathrm{rms}$. To achieve a contrast of $10^{-10}$ this level becomes extremely challenging: $0.01 \mathrm{~nm}$ rms.

- An increase of the telescope size does not significantly change the result and the same limitation holds for a $30 \mathrm{~m}$ and a $100 \mathrm{~m}$ telescope as long as the static aberrations originate from the instrument rather than the primary mirror. However, a larger telescope will result in a smaller integration time.

- Beyond the limitation of static aberrations, the signal to noise ratio depends on the level of the PSF halo. Therefore, the performance of AO systems for ELTs is a critical point which must be addressed thoroughly.

- Contrary to previous studies, it is shown that the feasibility of Earth-like planet detection with ELTs is questionable, for the reasons mentioned herein.

The present study did not take into account telescope pupil segmentation and obscuration, the pointing of the source on the coronagraph, the alignment of the pupil on the Lyot stop and the defects of the coronagraph (for instance chromatism). Background noise and Flat Fielding will be also critical. An actual system must consider all these sources of error to derive realistic performances as was done for the case of MIRI on JWST (Baudoz et al. 2005) and VLT Planet Finder (Beuzit \& et al. 2004). Scintillation is another source of error to consider in the case of large ground-based telescopes when dealing with a $10^{-10}$ level.

The detection (and even more the characterization) of Earth-like planets from the ground with Extremely Large Telescopes will be extremely challenging. A thorough analysis of the system as a whole (telescope $+\mathrm{AO}$ system + instrument) is strongly recommended to tackle the many and probably unknown sources of error.

\section{References}

Andersen, T., Ardeberg, A., \& Owner-Petersen, M. 2003, Euro50: Design Study of a $50 \mathrm{~m}$ adaptive Optics Telescope, Tech. rep.

Angel, R. 2003, in Towards Other Earths: DARWIN/TPF and the Search for Extrasolar Terrestrial Planets, ESA SP-539, 221

Baba, N., \& Murakami, N. 2003, PASP, 115, 1363

Baudoz, P., Rabbia, Y., \& Gay, J. 2000, A\&AS, 141, 319

Baudoz, P., Boccaletti, A., Riaud, P., et al. 2005, PASP, in press

Beuzit, J.-L., et al. 2004, VLT-PF Concept Feasibility Study, proposal to ESO, Tech. rep.

Bloemhof, E. E. 2004, ApJ, 610, L69

Bloemhof, E. E., Dekany, R. G., Troy, M., \& Oppenheimer, B. R. 2001, ApJ, 558, L71

Boccaletti, A. 2004, in Astronomy with High Contrast Imaging, EAS Pub. Ser., 12, 165

Boccaletti, A., Riaud, P., \& Rouan, D. 2002, PASP, 114, 132

Chelli, C. 2005, A\&A, in press

Close, L. M., Lenzen, R., Biller, B., Brandner, W., \& Hartung, M. 2005, in Science with Adaptive Optics, ESO Astrophysics Symposia, 46

Conan, R., Fusco, T., \& Rousset, G. 2005, in Science with Adaptive Optics, ESO Astrophysics Symposia, 97

Duparré, A., Ferre-Borrull, J., Gliech, S., et al. 2002, Appl. Opt., 41, 154

Gilmozzi, R. 2004, in Proc. SPIE, 5489, 1

Guyon, O. 2004, ApJ, 615, 562

Johns, M., Angel, J. R., Shectman, S., et al. 2004, in Proc. SPIE, 5489, 441

Jolissaint, L., \& Veran, J. 2002, in Beyond conventional adaptive optics, ESO Conference and Workshop Proceedings, 58, 201

Kuchner, M. J., \& Traub, W. A. 2002, ApJ, 570, 900

Lardiere, O., Salinari, P., Jolissaint, L., et al. 2004, in Proc. SPIE, 5382, 550

Marois, C., Doyon, R., Nadeau, D., et al. 2005, PASP, accepted [arXiv:astro-ph/0504652]

Marois, C., Doyon, R., Nadeau, D., Racine, R., \& Walker, G. A. 2003, in Astronomy with High Contrast Imaging, EAS Pub. Ser., 8, 233

Marois, C., Doyon, R., Racine, R., \& Nadeau, D. 2000, PASP, 112, 91

Nelson, J., Bolte, M., Abraham, R., et al. 2005, Thirty Meter Telescope Science-Based Requirements Document (SRD), Tech. rep.

Perrin, M. D., Sivaramakrishnan, A., Makidon, R. B., Oppenheimer, B. R., \& Graham, J. R. 2003, ApJ, 596, 702

Racine, R., Walker, G. A., Nadeau, D., Doyon, R., \& Marois, C. 1999, PASP, 111, 587

Rigaut, F., Veran, J.-P., \& Lai, O. 1998, in Proc. SPIE, 3353, 1038

Roddier, F., \& Roddier, C. 1997, PASP, 109, 815

Rouan, D., Riaud, P., Boccaletti, A., Clénet, Y., \& Labeyrie, A. 2000, PASP, 112, 1479

Sivaramakrishnan, A., Lloyd, J. P., Hodge, P. E., \& Macintosh, B. A. 2002, ApJ, 581, L59 